\title{
EFFECTS OF THE NEUTRINO MASS SPLITTING ON THE NONLINEAR MATTER POWER SPECTRUM
}

\author{
Christian Wagner ${ }^{1}$, Licia Verde ${ }^{1,2}$, and Raul Jimenez ${ }^{1,2}$ \\ ${ }^{1}$ Institut de Ciencies del Cosmos (ICC), Universitat de Barcelona (IEEC-UB), Marti i Franques 1, E08028 Barcelona, Spain \\ 2 ICREA-Institució Catalana de Recerca i Estudis Avançats \\ Received 2012 March 26; accepted 2012 May 15; published 2012 June 5
}

\begin{abstract}
We have performed cosmological $N$-body simulations which include the effect of the masses of the individual neutrino species. The simulations were aimed at studying the effect of different neutrino hierarchies on the matter power spectrum. Compared to the linear theory predictions, we find that nonlinearities enhance the effect of hierarchy on the matter power spectrum at mildly nonlinear scales. The maximum difference between the different hierarchies is about $0.5 \%$ for a sum of neutrino masses of $0.1 \mathrm{eV}$. Albeit this is a small effect, it is potentially measurable from upcoming surveys. In combination with neutrinoless double- $\beta$ decay experiments, this opens up the possibility of using the sky to determine if neutrinos are Majorana or Dirac fermions.
\end{abstract}

Key words: large-scale structure of universe - methods: numerical - neutrinos

Online-only material: color figures

\section{INTRODUCTION}

Neutrino oscillation experiments have demonstrated that neutrinos have non-zero mass (Fukuda et al. 1998). Measurements of the flavor changing oscillations have provided a difference in the squares of the masses of the lightest and heaviest mass eigenstates $\Delta m^{2} \simeq(0.05 \mathrm{eV})^{2}$, yielding a lower limit on the total neutrino mass $\left(\Sigma=\sum m_{v_{i}}\right)$. Ongoing and forthcoming ground-based neutrino experiments (Cremonesi 2010) are sensitive to neutrino flavor and to the nature of neutrino mass (Dirac or Majorana) but are only sensitive to the absolute mass scale for large masses. On the other hand, cosmological probes are blind to flavor but sensitive to the absolute neutrino mass scale and recently there has been significant progress in constraining the sum of neutrino masses from cosmological observations. Massive neutrinos affect the observed matter power spectrum: their free-streaming damps the small-scale power and modifies the shape of the matter power spectrum below the free-streaming length (Doroshkevich et al. 1980; Hu et al. 1998; Takada et al. 2006; Kiakotou et al. 2008). The cosmological constraints on the total neutrino mass are getting tighter - the current limits on the total mass are $\lesssim 0.3 \mathrm{eV}$ (e.g., Thomas et al. 2009; Reid et al. 2010a; Komatsu et al. 2011; Saito et al. 2011; Riemer-Sørensen et al. 2011; de Putter et al. 2012) - and closer to the experimental lower limits derived from accelerator, reactor, solar, and atmospheric neutrino oscillations (see the reviews by Lesgourgues \& Pastor 2006; Gonzalez-Garcia \& Maltoni 2008, and references therein). Detecting the effect of neutrino masses on cosmological structure and measuring the neutrino mass scale is well within the reach of upcoming cosmological surveys (e.g., Takada et al. 2006; Hannestad \& Wong 2007; Kitching et al. 2008; LSST 2009; Hannestad 2010; Lahav et al. 2010; Reid et al. 2010a; Jimenez et al. 2010; Carbone et al. 2011, and references therein).

The neutrino mass splitting required to explain the oscillation results implies that for three neutrino species there are two possible hierarchies: normal hierarchy $(\mathrm{NH})$ with two light states and one heavy state and a total mass $\Sigma \gtrsim 0.05 \mathrm{eV}$; and inverted hierarchy (IH) with two heavy states and one light state with $\Sigma \gtrsim 0.1 \mathrm{eV}$. If the absolute mass scale is much higher than the mass difference then the mass hierarchy does not matter (this is called degenerate spectrum). The degenerate hierarchy however is under pressure from observations (Thomas et al. 2009; Reid et al. 2010a; Gonzalez-Garcia et al. 2010); see Figure 1, where we have introduced the neutrino mass splitting parameter $\Delta$ relating the lightest $(m)$ and heaviest $(M)$ neutrino masses following Jimenez et al. $(2010)^{3}$

$$
\begin{aligned}
\mathrm{NH}: & \Sigma=2 m+M \quad \Delta=(M-m) / \Sigma \\
\mathrm{IH}: & \Sigma=m+2 M \quad \Delta=(m-M) / \Sigma .
\end{aligned}
$$

A determination of the hierarchy can complement results from neutrinoless double- $\beta$ decay to help determine the nature of the neutrino itself (Jimenez et al. 2010): Is it its own antiparticle? That is, is it a Majorana fermion? As discussed in Bahcall et al. (2004; see their Figure 3 recalling that a light neutrino mass of $0.07 \mathrm{eV}$ corresponds to $\Sigma \sim 0.2-0.25 \mathrm{eV}$ ), if the next generation of neutrinoless double- $\beta$ decay experiments find a signal, then neutrinos are Majorana. If these experiments do not see a signal, it is important to discriminate if that is because the signal is below the detection threshold, or if neutrinos are truly Dirac particles. Here is where cosmology enters (Jimenez et al. 2010): If $\Sigma>0.25 \mathrm{eV}$, then the hierarchy is effectively degenerate and neutrinos are Dirac. The interesting region to determine the hierarchy is for $0.1 \mathrm{eV}<\Sigma<0.25 \mathrm{eV}$, where the absence of neutrinoless double- $\beta$ decay indicates that neutrinos are Dirac only if the hierarchy is inverted. In Jimenez et al. (2010), we addressed the above question using linear theory to predict the effect of neutrinos on the matter power spectrum. Our conclusion was that an ambitious survey à la stage IV dark energy task force (Albrecht et al. 2006) could in principle distinguish between the IH and NH if $\Sigma<0.15 \mathrm{eV}$. However, because the effect on the matter power spectrum of neutrinos extends into the nonlinear regime, it is crucial to perform $N$-body simulations that include massive neutrinos to properly quantify the effect. The main physical effect that distinguishes different hierarchies is the fact that neutrinos of different masses have different transition from relativistic to non-relativistic, thus influencing the shape of the matter power

\footnotetext{
3 Since one mass splitting is much larger than the other, for cosmological applications we can safely ignore the small-mass splitting.
} 


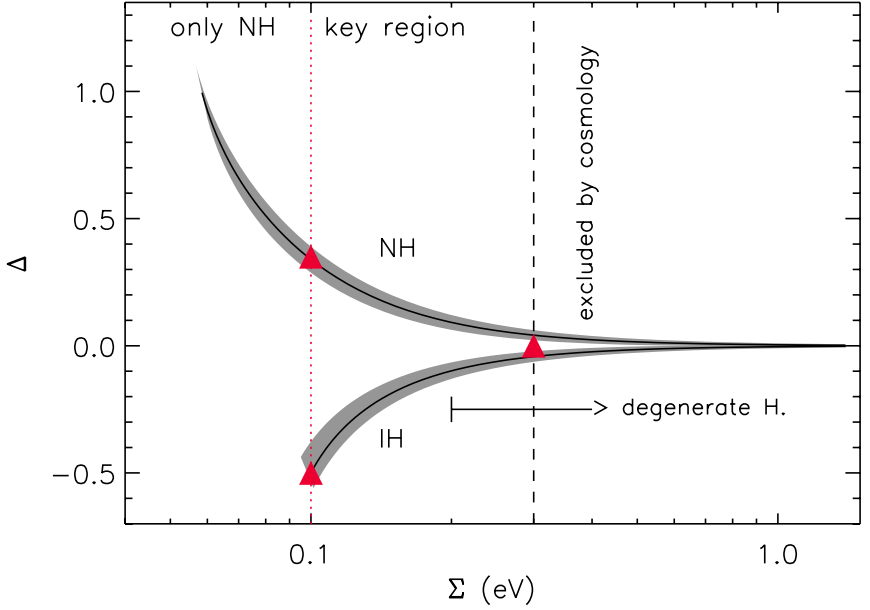

Figure 1. Constraints on the mass splitting from neutrino oscillations (shaded regions) and total neutrino mass from cosmology (vertical dashed line) in the parameter space defined by the sum of neutrino masses $\Sigma$ and the mass splitting parameter $\Delta$ characterizing the hierarchy. The key region where it is interesting to determine $\Delta$ is $0.1 \mathrm{eV}<\Sigma<0.3 \mathrm{eV}$. The triangles indicate the cases studied in this Letter: normal and inverted hierarchies (at $\Sigma=0.1 \mathrm{eV}$ ) and degenerate hierarchy (at $\Sigma=0.3 \mathrm{eV}$ ). Plot adapted from Figure 1 of Carbone et al. (2011). (A color version of this figure is available in the online journal.)

spectrum (Lesgourgues et al. 2004; Slosar 2006; de Bernardis et al. 2009). Agarwal \& Feldman (2011) performed $N$-body simulations for the IH and NH with $\Sigma=0.1 \mathrm{eV}$ by only altering the initial conditions. In contrast, in this Letter we present results for which the neutrino perturbations and their nonlinear evolution was fully taken into account during the simulation.

Note that neutrino oscillation experiments rule out large regions in the $\Sigma-\Delta$ parameter space (see Figure 1) and therefore it is worth investigating only the allowed region (gray swath in Figure 1).

\section{NUMERICAL SIMULATION METHOD}

In this Letter, we are interested in the effect of the neutrino mass splitting on the nonlinear matter power spectrum. To study this effect we run cosmological $N$-body simulations for different neutrino mass configurations: (1) three massless neutrinos; (2) three neutrinos of equal mass (degenerate case, $\Sigma=0.3 \mathrm{eV}$ ); (3) one massless neutrino and two massive neutrinos (IH, $\Sigma=0.1 \mathrm{eV}, \Delta=-0.50$ ); and (4) two light neutrinos and one heavy neutrino $(\mathrm{NH}, \Sigma=0.1 \mathrm{eV}, \Delta=0.32)$. When analyzing the simulation results, we focus on relative quantities like the ratio of the power spectrum for different neutrino mass configurations, since in this case systematic effects and sample variance are suppressed.

So far there have been mainly two approaches to incorporating neutrinos into cosmological $N$-body simulations: sampling the neutrino density with particles just like in the case of cold dark matter (White et al. 1983; Klypin et al. 1993; Brandbyge et al. 2008; Viel et al. 2010) or evolving the neutrino density on a grid (with a fixed size) using linear theory (Brandbyge \& Hannestad 2009; Viel et al. 2010). These two approaches were combined into a hybrid method by Brandbyge \& Hannestad (2010). The particle-based approach has the advantage of being able to capture the nonlinear evolution of the neutrinos and their effect on the cold matter components, while the linear evolution of the neutrino density on a grid is by construction only able to model the linear gravitational effect of the neutrinos on the nonlinear matter distribution. In the particle-based approach, however, the neutrinos are always treated as non-relativistic particles, since standard cosmological $\mathrm{N}$-body codes do not include relativistic effects. Another problem of this approach is that the finite number of particles used to sample the neutrino phase space generate shot noise. As the neutrinos have high thermal velocities, they move quickly away from their initial positions and give rise to a Poisson-like shot noise. Both these problems can be somewhat circumvented by starting the simulation at a sufficiently low redshift, when the neutrinos are practically non-relativistic and their input power spectrum is large compared to the shot noise. On the other hand, one does not want to start too late, when nonlinearities have already become significant on the scales of interest. Hence, in order to keep the shot noise sub-dominant already at the initial redshift of the simulation, a large number of neutrino tracers is required.

Studies by Brandbyge \& Hannestad (2010) and Bird et al. (2012) have shown that in spite of the shortcomings mentioned above the particle-based approach is more accurate in computing the effect of massive neutrinos on the nonlinear matter power spectrum than the grid-based approach. Hence, in this Letter, we present results of particle-based simulations only.

We focus on scales in the range of $0.01 h \mathrm{Mpc}^{-1}<k<$ $1 h \mathrm{Mpc}^{-1}$. These scales will be probed to high accuracy by future surveys (e.g., Euclid ${ }^{4}$ ) and are much less affected by baryonic physics, ${ }^{5}$ which we neglect in this Letter, than smaller scales. Hence, we simulate a volume of $\left(600 h^{-1} \mathrm{Mpc}\right)^{3}$ with a particle load of 1 billion cold matter tracers and 2 billion neutrino tracers.

We adopt a flat $\Lambda \mathrm{CDM}$ cosmology with cosmological parameters compatible with current constraints. The primordial curvature power spectrum is specified by the scalar amplitude $\Delta_{\mathcal{R}}^{2}=$ $2.45 \times 10^{-9}$ at the pivot scale $k_{p}=0.002 \mathrm{Mpc}^{-1}$ with a spectral index $n_{s}=0.97$. We keep the present-day total matter fraction the same for all neutrino models: $\Omega_{m}=\Omega_{\mathrm{CDM}}+\Omega_{v}+\Omega_{b}=0.27$ with the baryon fraction and the neutrino fraction given by $\Omega_{b}=0.046$ and $\Omega_{v}=\Sigma /\left(93.8 \mathrm{eVh} h^{2}\right)$, where $\Sigma$ is the sum of neutrino masses in units of $\mathrm{eV}$. We choose the Hubble parameter to be $h=0.7$. This choice of cosmological parameters yields a present-day linear mass variance in spheres of $8 h^{-1} \mathrm{Mpc}$ of $\sigma_{8} \approx 0.8$.

To set up the initial conditions and assessing the $\mathrm{N}$-body simulation results, we need accurate linear predictions for the transfer functions for each component of the universe. We modified the linear Einstein-Boltzmann solver CAMB (version 2012 January; Lewis et al. 2000) to output a separate transfer function for each neutrino species in addition to the individual linear transfer functions for cold dark matter, baryons, and photons.

To reduce transients due to the late start of the simulation (Scoccimarro 1998), we implement second-order Lagrangian perturbation theory for the cold matter component. We compute numerically the ( $k$-dependent) linear growth rate from two CAMB outputs around the initial redshift. The second-order growth function $D_{2}$ is approximated by $D_{2} \approx-3 / 7 D_{1}^{2}$, where $D_{1}$ is the linear growth function (Bouchet et al. 1995). At the initial redshift $\left(z_{i}=9\right)$ the neutrino perturbations are still very much in the linear regime. Hence, for the neutrino particles, it suffices to use the Zel'dovich approximation to displace

\footnotetext{
4 http://sci.esa.int/euclid

5 Recent cosmological hydrodynamical simulations including AGN feedback showed that baryonic effects alter the matter power spectrum by $1 \%, 10 \%$, and $30 \%$ at $k=0.1 h \mathrm{Mpc}^{-1}, k=1 h \mathrm{Mpc}^{-1}$, and $k=10 h \mathrm{Mpc}^{-1}$, respectively (van Daalen et al. 2011).
} 

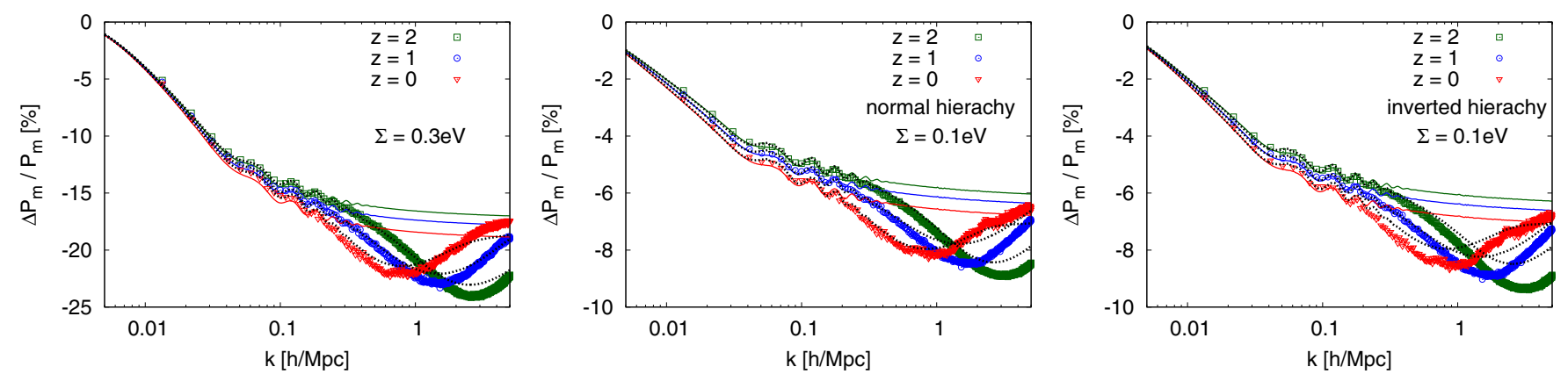

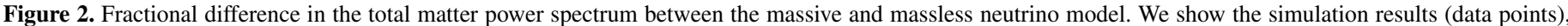

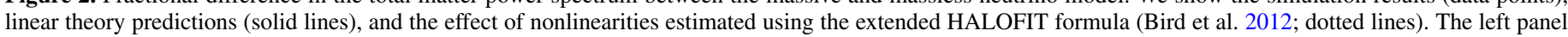

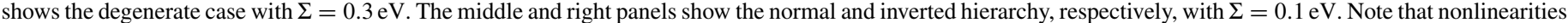
enhance the signal and lead to a maximum in the suppression located at mildly nonlinear scales.

(A color version of this figure is available in the online journal.)

the particles from the initial grid points and to assign the gravitational velocities. Then thermal velocities drawn from the appropriate Fermi-Dirac distribution are added to the neutrino velocities. We neglect higher-order multipoles of the neutrino phase space distribution (see Ma \& Bertschinger 1994 for a treatment of the full neutrino phase space). Tests with CAMB where these multipoles were set to zero at $z=z_{i}$ and then evolved further to low redshift have shown that the effect of these initial conditions on the linear matter power spectrum is negligible at $z \leqslant 2$ (R. de Putter 2012, private communication).

The simulations were carried out with the $N$-body code Gadget-2 (Springel 2005), which we modified to take into account the effect of the massive neutrinos (and the radiation) on the evolution of the scale factor $a$.

Using smaller test runs, we performed convergence tests on the neutrino time stepping. We found that a maximum time step $\max (\Delta \ln a)=0.025$ in the long-range particle-mesh force computation is sufficient for an accurate computation of the effect of the neutrinos on the matter power spectrum. Note that we disabled the Courant condition for the neutrino tracers. Hence, the time step for the long-range force is determined from the velocity dispersion of the cold matter tracers alone. This speeds up the computations significantly and leaves the nonlinear matter power spectrum virtually unchanged. We set the softening length of the short-range force to $20 \mathrm{kpc}^{-1}$ for both the cold matter and neutrino component.

Varying the initial redshift and the number of neutrino tracers, we confirmed that the measured ratio of the nonlinear matter power spectrum from simulations with and without massive neutrinos is robust against the neutrino shot noise and the residual transients due to the late start (Brandbyge et al. 2008).

We compute the total matter density contrast $\delta$ by assigning the particles to a $2048^{3}$ grid using the cloud-in-cell scheme. In this process, cold matter and neutrino particles are weighted by the fraction they contribute to $\Omega_{m}$. Using fast Fourier transforms and averaging $\left|\delta_{k}\right|^{2}$ over spherical shells with a bin width of $\Delta k=0.01 h \mathrm{Mpc}^{-1}$, we obtain the power spectrum $P(k)=\left\langle\left|\delta_{k}\right|^{2}\right\rangle$. Note that we only consider the non-relativistic neutrino species sampled by particles, i.e., the fluctuations in the radiation (relativistic neutrinos and photons) are not taken into account. At the redshifts of interest $(z \lesssim 2)$, however, radiation contributes a negligible amount to the total energy budget. One possible uncertainty in our simulation results is the shot noise coming from the cold matter particles. As the particles start off from a grid, the shot noise is sub-Poissonian and scale dependent at high redshifts. However, for $z \leqslant 1$ and $k<1 h \mathrm{Mpc}^{-1}$, even a Poisson shot noise is negligible. Hence, we do not attempt to correct for shot noise.

\section{RESULTS}

First, we compare the results of the degenerate case $(\Sigma=$ $0.3 \mathrm{eV}$ ) with previous works. We consider the fractional difference in the total matter power spectrum between models with and without massive neutrinos. One advantage of this relative quantity is that the sample variance present in the $\mathrm{N}$-body simulations cancels out almost completely. The result in the degenerate case is shown in the left panel of Figure 2. The simulation results show that nonlinearities enhance the effect on mildly nonlinear scales. This behavior is anticipated by perturbation theory (Saito et al. 2008; Wong 2008; Lesgourgues et al. 2009). In contrast to the linear theory predictions (solid lines), in the nonlinear case there is a maximum suppression, whose depth and position in $k$ depend on redshift. These numerical results are in excellent agreement with Brandbyge et al. (2008) and Viel et al. (2010). Using $N$-body simulations, Bird et al. (2012) modified HALOFIT (Smith et al. 2003) to model the ratio of the nonlinear matter power spectrum with and without massive neutrinos (black dotted lines). ${ }^{6}$

In the middle and right panels of Figure 2, we show the power spectrum suppression for the $\mathrm{NH}$ and $\mathrm{IH}$ with $\Sigma=0.1 \mathrm{eV}$. We observe the same qualitative behavior as before. Note that the small differences between the two hierarchies are not captured by the HALOFIT formula, which was fitted to $N$-body simulations for the degenerate hierarchy only. In order to make the small difference between the two models visible, the fractional difference in the total matter power spectrum between the two cases is shown in Figure 3. Similar to the case of massive versus massless neutrinos, the nonlinear evolution enhances the difference between the two models on mildly nonlinear scales (to a maximum suppression of $\sim 0.5 \%$ ). On even smaller scales which are in the stable clustering regime, the strong nonlinearities eventually overcome the initial differences and the remaining effect decreases with $k$ and may eventually drop below the linear theory prediction at large $k$. These results are in good agreement with Figure 8 of Agarwal \& Feldman (2011) and thereby confirm that their approximations are valid for a small total neutrino mass $\Sigma=0.1 \mathrm{eV}$.

\footnotetext{
6 Note that Bird et al. (2012) fitted the HALOFIT parameters mostly to a simulation suite with a much smaller box size $\left(150 \mathrm{Mpc} h^{-1}\right)$. The power spectrum suppression in those small-box simulations is about $1 \%$ smaller than in their large-box (512 $\left.\mathrm{Mpc} h^{-1}\right)$ simulations (see their Figure 4.).
} 


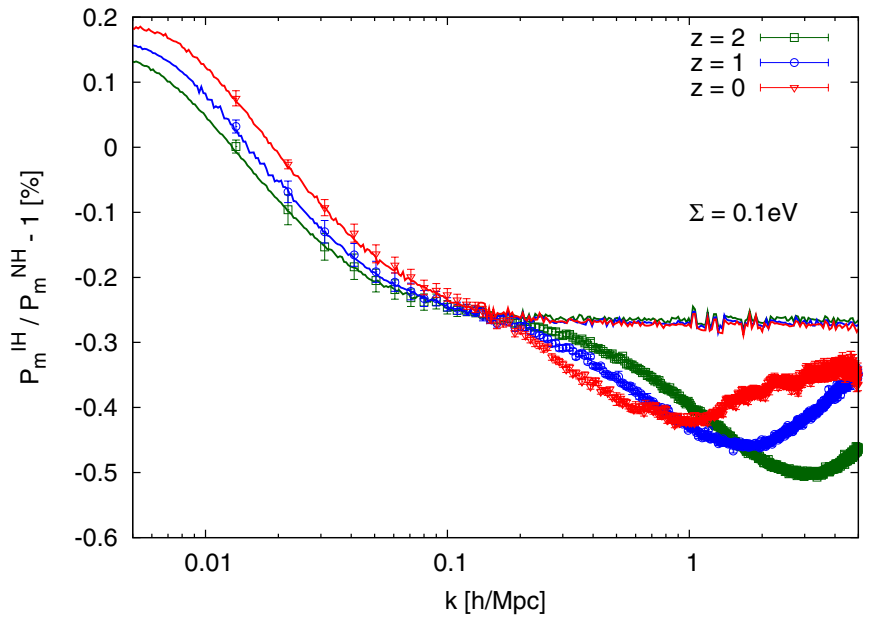

Figure 3. Fractional difference in the total matter power spectrum between the inverted and normal hierarchy run (the sum of neutrino masses is kept fixed and only the mass splitting is varied). Note that also in this case, nonlinearities enhance the effect on mildly nonlinear scales compared to linear theory predictions (solid lines). The error bars are estimated from the distribution in each bin.

(A color version of this figure is available in the online journal.)

For completeness, we show the neutrino power spectra for the $\mathrm{NH}$ and $\mathrm{IH}$ in Figure 4 . On large scales $\left(k \lesssim 0.1 h \mathrm{Mpc}^{-1}\right)$, the neutrino power spectra from the simulations agree well with the linear predictions. As expected, even at high redshift we observe a Poisson-like shot noise due to the large thermal velocities of the neutrinos. Although this shot noise is large in the neutrino power spectrum, it is much smaller when one considers the total matter density, since neutrinos contribute less than $1 \%$ to the total matter fraction. Additionally, on scales in the nonlinear regime, the matter power spectrum is dominated by the nonlinear power which is sourced from large-scale modes, for which shot noise is negligible.

\section{DISCUSSION AND CONCLUSIONS}

The main result of our numerical experiments is that nonlinearities enhance the dependence of the power spectrum on the different neutrino hierarchies, thus making the observational signature more pronounced. We estimate that, if all other cosmological parameters are known (including the sum of neutrino masses $\Sigma$ ), the two hierarchies can be distinguished with confidence for $\Sigma=0.1 \mathrm{eV}$, as illustrated in Figure 5, making the effect potentially measurable. We have assumed an effective

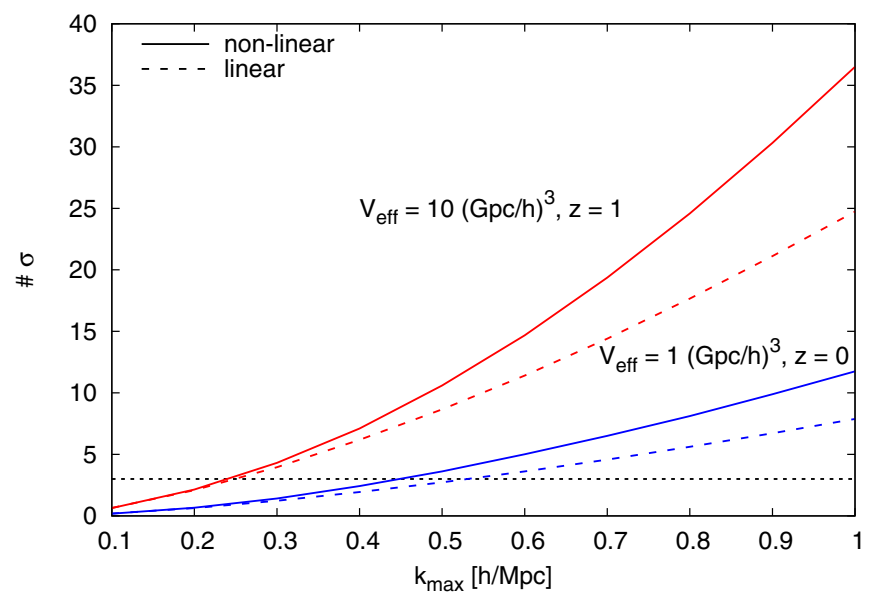

Figure 5. Forecast of the number of standard deviations separating the two hierarchies for $\Sigma=0.1 \mathrm{eV}$ as a function of the maximum $k$ vector considered and for two effective volumes- 1 and $10\left(\mathrm{Gpc} h^{-1}\right)^{3}-$ at $z=0$ and $z=1$, respectively. We have assumed that all other cosmological parameters are known. The horizontal dotted line indicates the $3 \sigma$ level.

(A color version of this figure is available in the online journal.)

volume of $1\left(\mathrm{Gpc} h^{-1}\right)^{3}$ at $z=0$ (blue) and $10\left(\mathrm{Gpc} h^{-1}\right)^{3}$ at $z=1$ (red). ${ }^{7}$

Whether degeneracies with other cosmological parameters and systematic effects (galaxy bias, baryonic physics, observational limitations, etc.) will cancel the detectability of the effect remains to be explored and will be considered elsewhere (C. Wagner et al. 2012, in preparation).

Our findings indicate that cosmology has the potential of determining the neutrino hierarchy-not only for $\Sigma<0.1 \mathrm{eV}$, for which only the NH is possible-in the interesting window $0.1<\Sigma \lesssim 0.25 \mathrm{eV}$.

As an aside and already noted in Jimenez et al. (2010), cosmology is more sensitive to $|\Delta|$ than to its sign: a measurement of $|\Delta|$ in agreement with that predicted by oscillations experiments for the measured $\Sigma$ would provide a convincing consistency check for the total neutrino mass constraint from cosmology.

However, it is very important to keep in mind that one needs theoretical predictions of the absolute nonlinear power spectrum with an uncertainty smaller than the signal (i.e., $0.1 \%$ ), to actually be able to make any measurement of the neutrino hierarchy. In this Letter, we presented numerical predictions only

\footnotetext{
7 These volumes roughly correspond to the volume out to $z=0.5$ and between $z=0.5$ and $z=1.5$ in $1 / 10$ of the sky, respectively, in a standard $\Lambda \mathrm{CDM}$ universe.
}
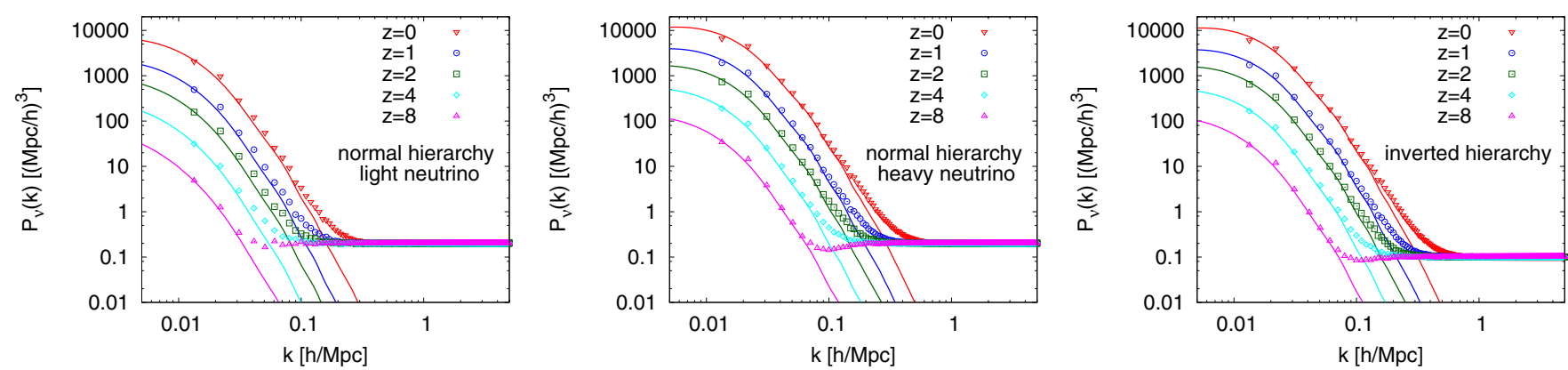

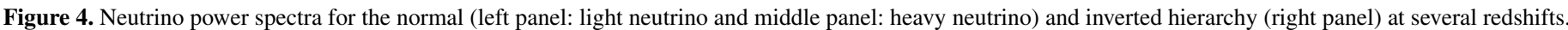

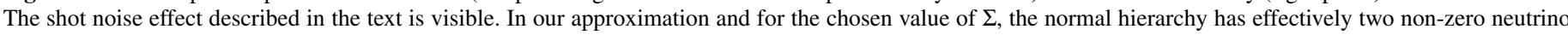
masses ( $M$ and $m$ in the notation of Equation (1)), while the inverted hierarchy has effectively only one massive eigenstate $M$ (the light neutrino is massless).

(A color version of this figure is available in the online journal.) 
for the relative nonlinear power spectrum suppression. This relative quantity is much more robust against numerical errors. Even without massive neutrinos, it is challenging to compute the nonlinear power spectrum to sub-percent precision (e.g., Heitmann et al. 2010). On small scales ( $\left.k \gtrsim 1 h \mathrm{Mpc}^{-1}\right)$, baryon physics, which is strongly model dependent and computationally very intensive, is non-negligible and makes it very hard to achieve this accuracy in the foreseeable future (e.g., van Daalen et al. 2011). In addition, although it is in principle much less demanding to compute the linear power spectrum to high accuracy, different linear Einstein-Boltzmann codes (e.g., CAMB; Lewis et al. 2000 and CLASS; Blas et al. 2011) still do not agree to $0.1 \%$ precision on the relevant scales.

Despite these very challenging and open problems, precision measurements of the large-scale structure of the universe remain an interesting avenue to determine the neutrino hierarchy.

We acknowledge the participation of Carlos Peña-Garay at an early stage of this project and thank him for many interesting discussions. C.W. is grateful for insightful discussions with Roland de Putter. C.W. and L.V. are supported by FP7-IDEASPhys.LSS 240117. L.V. and R.J. are supported by FPA201129678-C02-02.

\section{REFERENCES}

Agarwal, S., \& Feldman, H. A. 2011, MNRAS, 410, 1647

Albrecht, A., Bernstein, G., Cahn, R., et al. 2006, arXiv:astro-ph/0609591

Bahcall, J. N., Murayama, H., \& Pena-Garay, C. 2004, Phys. Rev. D, 70, 033012

Bird, S., Viel, M., \& Haehnelt, M. G. 2012, MNRAS, 420, 2551

Blas, D., Lesgourgues, J., \& Tram, T. 2011, J. Cosmol. Astropart. Phys., JCAP07(2011)034

Bouchet, F. R., Colombi, S., Hivon, E., \& Juszkiewicz, R. 1995, A\&A, 296, 575

Brandbyge, J., \& Hannestad, S. 2009, J. Cosmol. Astropart. Phys., JCAP05(2009)002

Brandbyge, J., \& Hannestad, S. 2010, J. Cosmol. Astropart. Phys., JCAP01(2010)021

Brandbyge, J., Hannestad, S., Haugbølle, T., \& Thomsen, B. 2008, J. Cosmol. Astropart. Phys., JCAP08(2008)020

Carbone, C., Verde, L., Wang, Y., \& Cimatti, A. 2011, J. Cosmol. Astropart. Phys., JCAP03(2011)030

Cremonesi, O. 2010, arXiv:1002.1437 de Bernardis, F., Kitching, T. D., Heavens, A., \& Melchiorri, A. 2009, Phys Rev. D, 80, 123509

de Putter, R., Mena, O., Giusarma, E., et al. 2012, arXiv:1201.1909

Doroshkevich, A. G., Zel'dovich, Ya. B., Syunyaev, R. A., \& Khlopov, M. Yu. 1980, SvA Lett., 6, 252

Fukuda, Y., Hayakawa, T., Ichihara, E., et al. 1998, Phys. Rev. Lett., 81, 1562

Gonzalez-Garcia, M., \& Maltoni, M. 2008, Phys. Rept., 460, 1

Gonzalez-Garcia, M. C., Maltoni, M., \& Salvado, J. 2010, J. High Energy Phys., 2010, 117

Hannestad, S. 2010, Prog. Part. Nucl. Phys., 65, 185

Hannestad, S., \& Wong, Y. Y. Y. 2007, J. Cosmol. Astropart. Phys., JCAP07(2007)04

Heitmann, K., White, M., Wagner, C., Habib, S., \& Higdon, D. 2010, ApJ, 715, 104

Hu, W., Eisenstein, D. J., \& Tegmark, M. 1998, Phys. Rev. Lett., 80, 5255

Jimenez, R., Kitching, T., Pena-Garay, C., \& Verde, L. 2010, J. Cosmol. Astropart. Phys., JCAP05(2010)035

Kiakotou, A., Elgaroy, O., \& Lahav, O. 2008, Phys. Rev. D, 77, 063005

Kitching, T. D., Heavens, A. F., Verde, L., Serra, P., \& Melchiorri, A. 2008, Phys. Rev. D, 77, 103008

Klypin, A., Holtzman, J., Primack, J., \& Regos, E. 1993, ApJ, 416, 1

Komatsu, E., Smith, K. M., Dunkley, J., et al. 2011, ApJS, 192, 18

Lahav, O., Kiakotou, A., Abdalla, F. B., \& Blake, C. 2010, MNRAS, 405, 168

Lesgourgues, J., Matarrese, S., Pietroni, M., \& Riotto, A. 2009, J. Cosmol. Astropart. Phys., JCAP06(2009)017

Lesgourgues, J., Pastor, S., \& Perotto, L. 2004, Phys. Rev. D, 70, 045016

Lesgourgues, J., \& Pastor, S. 2006, Phys. Rept., 429, 307

Lewis, A., Challinor, A., \& Lasenby, A. 2000, ApJ, 538, 473

LSST Science Collaborations, et al. 2009, arXiv:0912.0201

Ma, C.-P., \& Bertschinger, E. 1994, ApJ, 429, 22

Reid, B. A., Verde, L., Jimenez, R., \& Mena, O. 2010a, J. Cosmol. Astropart. Phys., JCAP01(2010)003

Riemer-Sørensen, S., Blake, C., Parkinson, D., et al. 2011, Phys. Rev. D, 85, 081101

Saito, S., Takada, M., \& Taruya, A. 2008, Phys. Rev. Lett., 100, 191301

Saito, S., Takada, M., \& Taruya, A. 2011, Phys. Rev. D, 83, 043529

Scoccimarro, R. 1998, MNRAS, 299, 1097

Slosar, A. 2006, Phys. Rev. D, 73, 123501

Smith, R. E., Peacock, J. A., Jenkins, A., et al. 2003, MNRAS, 341, 1311

Springel, V. 2005, MNRAS, 364, 1105

Takada, M., Komatsu, E., \& Futamase, T. 2006, Phys. Rev. D, 73, 083520

Thomas, S. A., Abdalla, F. B., \& Lahav, O. 2009, Phys. Rev. Lett., 105, 031301

van Daalen, M. P., Schaye, J., Booth, C. M., \& Dalla Vecchia, C. 2011, MNRAS, 415,3649

Viel, M., Haehnelt, M. G., \& Springel, V. 2010, J. Cosmol. Astropart. Phys., JCAP06(2010)015

White, S. D. M., Frenk, C. S., \& Davis, M. 1983, ApJ, 274, L1

Wong, Y. Y. Y. 2008, J. Cosmol. Astropart. Phys., JCAP10(2008)035 